\title{
Automated Image Alignment and Distortion Removal for 3-D Serial Sectioning with Electron Backscatter Diffraction
}

\author{
Amanda J. Levinson ${ }^{1}$, David J. Rowenhorst ${ }^{1}$, Alexis C. Lewis ${ }^{2}$ \\ ${ }^{1}$ Naval Research Laboratory, 4555 Overlook Avenue SW, Washington, DC 20375 \\ ${ }^{2}$ Formerly Naval Research Laboratory, 4555 Overlook Avenue SW, Washington, DC 20375
}

Three-dimensional characterization is required for measuring the true shape, connectivity, and spatial distribution of microstructural features. For accurate property or processing correlations with 3D microstructures, a statistically significant volume of data is required. Serial sectioning has emerged as pertinent method to obtain large volumes of 3D structural information while maintaining a relatively high spatial resolution [1]. For this process, a large series of 2D images are collected on the same area in successive parallel planes then reconstructed to form the 3D volume. Focused Ion Beam (FIB) sectioning in conjunction with SEM imaging excels for the characterization of fine features $(<5 \mu \mathrm{m})$, but lacks the milling rate to collect large statistically relevant volumes of material. Serial sectioning, using mechanical polishing or alternative milling methods [2] for material removal is capable of collecting much larger volumes, with fields of view up to a several $\mathrm{mm}^{2}$ while imaging with resolutions better than $1 \mu \mathrm{m}$, allowing it to operate on a length scale relevant for a large number of material problems.

The biggest limitation to any 3D characterization is typically the image processing and segmentation of relevant structures. However, Electron backscatter diffraction (EBSD) is a powerful characterization tool that maps the orientation on the surface of a crystalline material and is an attractive imaging tool for serial sectioning polycrystalline samples. EBSD data is based on a physical attribute of the material, the crystallographic orientation at every point, thus eliminating lengthy semi-automated data segmentation procedures associated with other imaging. However, the experimental setup requiring a high sample tilt angle of $70^{\circ}$ often results in significant geometric image distortions [3], which can be variable from section to section due to small sample orientation changes when the sample is loaded and unloaded from the SEM chamber between each section. Furthermore, these distortions can be exacerbated as the scanning area increases at low magnifications.

In this investigation a large volume of $316 \mathrm{~L}$ stainless steel has been collected via serial sectioning using ex-situ mechanical polishing with EBSD imaging to evaluate grain boundary networks as a function of processing. Each 2D section is composed of a grid of overlapping EBSD images that span a total area of $1 \mathrm{~mm}^{2}$ with an in-plane resolution of $0.5 \mu \mathrm{m}$ and a section-to-section spacing of approximately $1 \mu \mathrm{m}$. After montaging the EBSD images for each section, large and inconsistent image distortions are present between sections, as shown in Figure 1(a) by the difference image of two sequential sections. It was found that the distortion in each section is unique due to the changing experimental parameters, such as sample placement and SEM conditions from the ex-situ nature of the technique.

An automated procedure has been developed for eliminating the distortions within each 2D section individually without requiring a distortion calibration for every possible imaging condition a priori. The procedure utilizes another imaging technique that is undistorted, but lacks the microstructural information needed for reconstruction as a "ground truth" image for the geometry in each section. In this case optical microscopy images of fiducial marks on the perimeter of the analysis area were used as the optical micrographs were found to have virtually no significant distortions over a wide field-of-view. 
Each EBSD section was undistorted to align the fiducuial marks in the EBSD image with its corresponding optical image using a Nelder-Mead optimization technique with mutual information (MI) as the figure of merit. MI is a quantitative measure of the information two variables share; this value is maximized when the EBSD and optical images are perfectly aligned. MI has two benefits. First, it can be used to align multimodal data, i.e. images collected using different techniques. Second, it has a high tolerance for convergence, ensuring that the optimization has reached a local minimum solution. After this step, all undistorted EBSD sections are aligned in the same manner to each other. Figure 1(b) shows the difference image of the same two sections in 1(a) after final alignment.

This automated process was applied to the reconstruction of 46 sections of $316 \mathrm{~L}$ composing a volume of approximately $825 \times 825 \times 46 \mu \mathrm{m}$, containing just over 20,000 grains (Figure 2 shows a tenth of the reconstructed volume). By comparison, this volume is about 100 times larger than a typical volume obtained via FIB sectioning. The major benefit of utilizing these tools for the reconstruction of large 3D volumes is that image distortions do not need to be measured directly, but are accounted for through image correlation techniques. [4]

[1] M.D. Uchic, M.A. Groeber, and A.D. Rollett, JOM 63 (2011) p. 25.

[2] M.P. Echlin, A. Mottura, C.J. Torbet and T.M. Pollock, Review of Scientific Instruments 83 (2012) p. 023701-1.

[3] G. Nolze, Ultramicroscopy 107 (2007) p. 172.

[4] This work was funded by the Naval Research Laboratory under the auspices of the Office of Naval Research and from the Structural Metallics program of ONR.

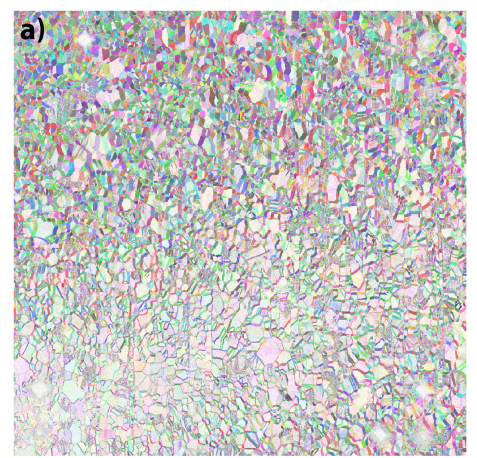

b)

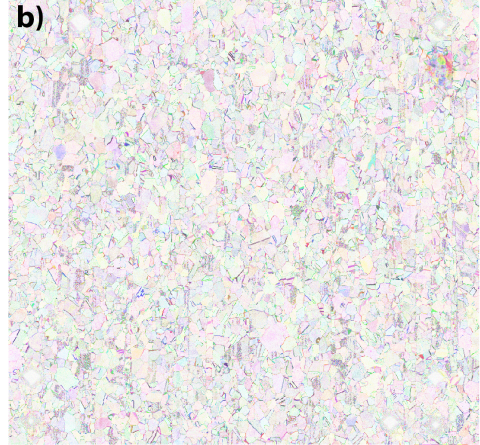

Figure 1. Difference map between two consecutive EBSD sections with (a) after stitching and (b) after alignment. White indicates minimal difference between the two images.

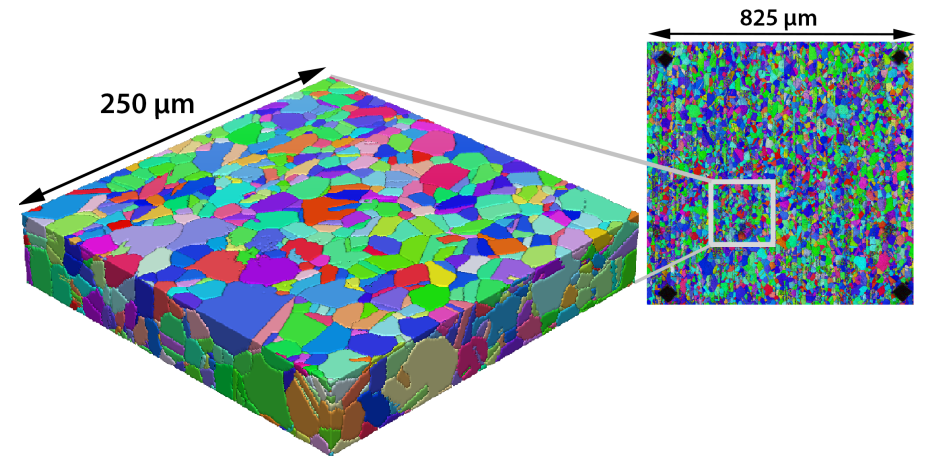

Figure 2. Reconstructed volume taken from the white square inset in the 2D section on right. 